\title{
Distances in spaces of physical models: partition functions versus spectra
}

\author{
Gunther Cornelissen $^{1}$ • Aristides Kontogeorgis ${ }^{2}$
}

Received: 25 February 2016 / Revised: 28 August 2016 / Accepted: 29 August 2016

Published online: 8 November 2016

(C) The Author(s) 2016. This article is published with open access at Springerlink.com

\begin{abstract}
We study the relation between convergence of partition functions (seen as general Dirichlet series) and convergence of spectra and their multiplicities. We describe applications to convergence in physical models, e.g., related to topology change and averaging in cosmology.
\end{abstract}

Keywords Zeta function · Partition function - Riemannian manifold - Spectrum • Convergence $\cdot$ Cosmological model

Mathematics Subject Classification $30 \mathrm{~B} 50 \cdot 58 \mathrm{C} 40 \cdot 83 \mathrm{~F} 05$

\section{Introduction}

In this paper, we study the relation between closeness of spectra of operators and of spectral partition functions. Spectra of operators play a central role in physical theories, as outcomes of a physical measurement. As motivation for our problem, we provide two examples where questions about closeness of spectra of operators (as describing specific physical models) come up naturally in cosmology.

The first one arises in connection with the averaging problem in cosmology (compare $[4,18]$ ) and the question of topology change under evolution of the universe. It is

Gunther Cornelissen

g.cornelissen@uu.nl

Aristides Kontogeorgis

kontogar@math.uoa.gr

1 Mathematisch Instituut, Universiteit Utrecht, Postbus 80.010, 3508 TA, Utrecht, The Netherlands

2 Department of Mathematics, National and Kapodistrian University of Athens, Panepistimioupolis, 15784 Athens, Greece 
clear that spectra contain topological information, cf. e.g., [14]. How to make mathematical sense (in terms of spectra) of such statements as "the universe is homogeneous on average", or "a sudden change of topology in the universe (e.g., from orientable to non-orientable) is unlikely?".

Seriu [17] proposes to use eigenvalues of the Laplace-Beltrami operator on spatial sections of a cosmological model to construct a metric on the space of such Riemannian manifolds up to some notion of "large scale isospectrality". He argues that the use of the plain difference of spectra as a measure is flawed: large energy contributions (corresponding to small-scale geometry) should carry a lower weight, and the dominant weight should be put on the small spectrum (corresponding to large-scale geometry). Therefore, he introduces a cut-off $N$ and introduces a metric that compares the first $N$ eigenvalues. Second, the eigenvalue difference is not a dimensionless quantity, and because of this, he suggests comparing quotients of spectra. However, as $N \rightarrow+\infty$, the distance he proposes diverges.

We argue in this paper that it is natural not to use a cut-off function, but to rather use a distance between the zeta functions (which, like partition functions, give more weight to low energy in their region of convergence), considered as complex functions; here, one may use classical notions of distance between complex functions [6] used in the study of limits of holomorphic or meromorphic functions. Also, the zeta functions are dimensionless. We explain this in Sect. 2.

The second set of examples relates to the use of eigenvalues as dynamical variables in gravity. Gravity coupled to matter can be given a spectral description using the framework of noncommutative geometry [5]. Even by ignoring the matter part, one arrives at an interesting description of classical gravity (general relativity) in terms of spectral data. These spectra form a diffeomorphism-invariant set of coordinates on the space of manifolds, up to isospectrality. Diffeomorphism invariant coordinates are an important prerequisite for certain programmes to quantize gravity (although 't Hooft [19] has argued that "deterministic quantum mechanics" might require a preferred coordinate frame that becomes irrelevant by information loss). In this way, spectra were used as dynamical variables for classical gravity by Landi and Rovelli [12]; see also [1].

The distance above (and its generalizations) provides a topology on the space of partition functions and it is a natural question to study the meaning of convergence of partition functions of models in terms of the spectrum itself. This is exactly what we study more abstractly: our Theorem 3.2 shows that it is a certain $\ell^{1}$-convergence of sequences of (suitable powers of) the eigenvalues. The same problem makes sense for generalized Dirichlet series/partition functions, and in the final sections of the paper, we study this problem from various perspectives, including via the Laplace-Stieltjes transform. At the end of Sect. 4, we give an example of how to describe convergence of eigenfunctions in terms of convergence of certain spectral zeta functions.

Finally, we remark that in recent years, Bayesian inference has been applied successfully to the comparison of cosmological models [11]. For example, Niarchou and Jaffe [15] have used cosmic microwave background radiation measurements to study the most likely topology of the universe. In brief, the Bayesian method investigates, under certain hypotheses, which is the most likely of a finite list of cosmological models (in [15], five different topologies) to match observational data (in [15], WMAP 
data). By contrast, the spectral distance seems a more theoretical tool, that can show how a characteristic of a model (e.g., in [17], orientability) is stable or unstable by a small perturbation of the model. It seems this is related to the discussion on the final page of [15] on the nature of the primordial power spectrum, and whether it is possible to keep the spectrum identical at small scales (matching observations), but perturbed at large scales (changing topology). Whereas in the Bayesian paradigm, one weighs finitely many possible points in the space of all models, spectral distances relate to navigating continuously in the space of all models.

\section{A spectral distance for cosmological models}

As we explained in the introduction, it is useful to have a metric between cosmological models. We will describe our construction for closed Riemannian spatial sections of Lorentzian spacetimes (it will work equally well for Euclidean gravity models, but not for metric of general signature). To compare spatial sections, one needs a metric between Riemannian manifolds. If $X$ is such a closed (= compact, no boundary) smooth Riemannian manifold with Laplace-Beltrami operator $\Delta_{X}$ and non-zero spectrum $\lambda_{1} \leq \lambda_{2} \leq \cdots$, its spectral zeta function is $\zeta_{X}(s):=\operatorname{Tr}\left(\Delta_{X}^{-s}\right)=\sum_{v \geq 1} \lambda^{-s}$. The spectral zeta function $\zeta_{X}$ converges absolutely for $\Re(s)>d / 2$, where $\bar{d}$ is the dimension of $X$ [16]. We propose the following function as a distance on suitable spaces of Riemannian geometries up to isospectrality:

Proposition 2.1 Let $\mathcal{M}$ denote a space of Riemannian manifolds up to isospectrality, with

$$
\sup \{\operatorname{dim}(X): X \in \mathcal{M}\}<2 \gamma
$$

finite. Then, for any $X_{1}, X_{2} \in \mathcal{M}$, the function

$$
d\left(X_{1}, X_{2}\right):=\sup _{\gamma<s<\gamma+1}|\log | \frac{\zeta_{X_{1}}(s)}{\zeta_{X_{2}}(s)}||
$$

where $\Re(s)>\gamma$ is a common plane of convergence for the spectral zeta functions of $X_{1}$ and $X_{2}$, defines a metric on $\mathcal{M}$.

Proof The function $d$ is positive, and if $d\left(X_{1}, X_{2}\right)=0$, then $\left|\zeta_{X_{1}}(s)\right|=\left|\zeta_{X_{2}}(s)\right|$ for all $s$ in the interval $] \gamma, \gamma+1$ [. Since this set has accumulation points, and since the zeta function is positive real for such values of $s$, we find that $\zeta_{X_{1}}=\zeta_{X_{2}}$ as complex functions. Hence, the identity theorem for Dirichlet series [10] implies that $X_{1}$ and $X_{2}$ are isospectral. The function $d$ is symmetric, since $\left|\log \left(x^{-1}\right)\right|=|\log (x)|$. Finally, the triangle inequality follows from $\zeta_{X_{1}}(s) / \zeta_{X_{3}}(s)=\zeta_{X_{1}}(s) / \zeta_{X_{2}}(s) \cdot \zeta_{X_{2}}(s) / \zeta_{X_{3}}(s)$ and the usual properties of the absolute value.

This is a distance that weighs correctly the energy contributions (large-scale geometries contribute more), but does not depend on a cut-off in the spectrum, nor diverges if a cut-off tends to infinity. 
Remark 2.2 Taking the supremum over $\gamma<s<\gamma+1$ is quite random: any set with an accumulation point and avoiding the poles of the zeta functions will do. Also, the distance $d$ can be replaced by $d /(1+d)$ to have it take values in the unit interval. The exact numerical values of the metric are not so relevant, but rather, their interrelation and the topology and uniformity that they induce.

Example 2.3 If $S_{r}$ denotes a circle of radius $r$, then $d\left(S_{r_{1}}, S_{r_{2}}\right)=4\left|\log \left(r_{1} / r_{2}\right)\right|$. This example shows that the distance can be non-differentiable in the parameter space of a family.

Example 2.4 Let us compute the spectral distance between a sphere $S$ and a real projective space $\mathbf{R} \mathbf{P}^{2}$ with the same volume $4 \pi$; this example was considered in [17] to argue that topological change of spatial slices during cosmological evolution is not unlikely. The zeta functions are

$$
\zeta_{S}=\sum_{\nu=1}^{\infty} \frac{2 v+1}{v^{s}(v+1)^{s}} \quad \text { and } \quad \zeta_{\mathbf{R P}^{2}}=\sum_{\nu=1}^{\infty} \frac{4 v+1}{v^{s}(2 v+1)^{s}}
$$

A numerical experiment suggests that the maximum in the distance formula is attained at $s=2$, and there we get $d\left(S, \mathbf{R P}^{2}\right)=\log \left(4-\pi^{2} / 3\right) \approx 0.342$.

Remark 2.5 A distance between Riemannian manifolds up to isometry was constructed by the first author and de Jong, who have, furthermore, given a spectral characterization of when a diffeomorphism of closed, smooth Riemannian manifolds is an isometry, in terms of equality of more general zeta functions under pullback by the map [7]. Also, this distance is based on a dimensionless object (zeta functions). However, such distance is much more involved than the one above (it uses infinitely many zeta functions), and the one above suffices from the spectral point of view.

The natural question arises as to what the relation is between convergence in the topology defined by this spectral distance and natural metrics in the space of values of the spectrum itself. The same problem exists for more general models based on spectra of operators as dynamical values (such as in the gravitational models discussed in the introduction, or statistical mechanical models). We recast this into a problem about general Dirichlet series, as follows: suppose that we have pointwise convergence of a sequence of general Dirichlet series

$$
D_{n}(s)=\sum_{\nu \geq 1} a_{n, \nu} e^{-s \mu_{n, v}} \rightarrow D(s)=\sum_{\nu \geq 1} a_{\nu} e^{-s \mu_{\nu}},
$$

all of which converge absolutely in a common half-plane $\Re(s)>\gamma$, with $a_{*}$ complex coefficients, and $\mu_{*}$ is a strictly increasing sequence of real numbers. Examples: if all coefficients $a_{v}$ are one, $D(s)$ is the partition function of a classical discrete canonical ensemble with total energy $\mu_{v}$ in the $\nu$-th microstate for $s=1 / k T$ ( $T$ temperature, $k$ Boltzmann constant); if all coefficients are integers, $D(s)$ is the partition function of a discrete quantum system whose Hamiltonian has eigenvalues $\mu_{v}$ with multiplicity $a_{v}$ (or a classical system with degeneracies), etc. 
In this paper, we study what this implies about "convergence" of the sequences $\left(a_{n, v}\right)$ and $\left(\mu_{n, v}\right)$. First, we consider the case of classical Dirichlet series, where $a_{*}=1$ and $\mu_{*}=\log \lambda_{*}$, and in Theorem 3.2, we prove that (1) is equivalent to the $\ell^{1}$ convergence of $\left(\lambda_{n, v}^{\gamma}\right)$ to $\left(\lambda_{v}^{\gamma}\right)$, for any real $\gamma$ strictly larger than the common abscissa of absolute convergence of the series $D_{n}$. In Theorem 4.3, we consider the case where $\mu_{n, v}=\mu_{v}$ is independent of $n$; then, for every $n,\left(a_{n, v}\right)$ converges to $\left(a_{n}\right)$. The most general case is studied in Sect. 5 from the point of view of the Perron's formula for Dirichlet series (where we give a concrete result under some hypotheses), and from the point of view of the Laplace-Stieltjes transform in Sect. 6, where we prove the equivalence to Lipschitz convergence of some step functions.

Specifically, if $\left\{X_{n}\right\}_{n=1}^{\infty} \cup\{X\}$ is a sequence of connected, closed, smooth Riemannian manifolds such that $d:=\sup \operatorname{dim} X_{n}$ is finite, convergence of their zeta functions implies convergence of their spectra in the sense of Theorem 3.2.

\section{Convergence of Dirichlet series}

Notation 3.1 Let us, once and for all, introduce the following convenient notation: if $s \in \mathbf{C}$ and $\Lambda=\left(\lambda_{v}\right)_{\nu=1}^{\infty}$ is a sequence of positive real numbers, we denote by $\Lambda^{s}$ the sequence $\left(\lambda_{v}^{s}\right)_{\nu=1}^{\infty}$.

\section{Theorem 3.2 Suppose that}

$$
D_{n}(s)=\sum_{\nu \geq 1} \lambda_{n, \nu}^{-s} \quad \text { and } \quad D(s):=\sum_{\nu \geq 1} \lambda_{\nu}^{-s}
$$

is a family of (generalized) Dirichlet series for $n=\emptyset, 1,2, \ldots$, where, for each $n$, $\Lambda_{n}:=\left(\lambda_{n, v}\right)_{\nu=1}^{\infty}$ forms a sequence of increasing positive real numbers with finite multiplicities. Assume that all series $D_{n}(s)$ are convergent in a common right halfplane $\mathfrak{R}(s)>\tilde{\gamma}>0$. Then, the following are equivalent:

(i) As $n \rightarrow+\infty$, the functions $D_{n}(s)$ converge to $D(s)$, pointwise in $s$ with $\Re(s)>$ $\tilde{\gamma}$

(ii) For every fixed $\nu$, any bounded subsequence of $\left\{\lambda_{n, v}\right\}_{n=1}^{\infty}$ converges to the same element $\lambda \in \Lambda$, and

$$
\#\left\{\left(\lambda_{n, v}\right)_{n=1}^{\infty}: \lim _{n \rightarrow \infty} \lambda_{n, v}=\lambda\right\}=\#\left\{\lambda_{v}: \lambda_{v}=\lambda\right\} .
$$

(iii) For some (equivalently, all) $\gamma>\tilde{\gamma}, \Lambda_{n}^{-\gamma}$ converges to $\Lambda^{-\gamma}$ in $\ell^{1}$.

Remark 3.3 The assumption that all series $D_{n}(s)$ are convergent in a common right half-plane $\Re(s)>\tilde{\gamma}$ is a minimal necessary assumption, since if this is not the case, the questions we ask are void.

The series $D_{n}$ (when divergent at $s=0$ ), converges for $\Re(s)>\gamma_{n}$, where (see Chapter 1, Section 6 of [10]):

$$
\gamma_{n}=\limsup _{\nu \rightarrow \infty} \frac{\log v}{\log \lambda_{n, v}} .
$$

The hypothesis says that $\tilde{\gamma}:=\sup \gamma_{n}$ is finite. 
Proof of Theorem 3.2 Since we later want to interchange some limits, we will first prove:

Lemma 3.4 The sequences $S_{n, N}(s):=\sum_{\nu=1}^{N} \lambda_{n, v}^{-s}$ converge to $D_{n}(s)$ for $N \rightarrow+\infty$ uniformly in $n$.

Proof of Lemma 3.4 The uniform convergence means that

$$
\forall \epsilon>0, \exists N_{0} \geq 1, \forall N>N_{0}, \quad\left|\sum_{\nu=1}^{N} \lambda_{n, \nu}^{-s}-D_{n}(s)\right| \leq \epsilon
$$

where $N_{0}$ does not depend on $n$.

Suppose $\Re(s)>\tilde{\gamma}$ is a common plane of convergence for all $D_{n}$, and set $\Re(s)=$ $\tilde{\gamma}+\delta$ for $\delta>0$. By Eq. (2), $\lambda_{n, v} \geq \tilde{\gamma} \bar{\nu}$. Hence, the tails of $D_{n}$ are bounded as follows:

$$
\sum_{\nu=N+1}^{\infty} \lambda_{n, \nu}^{-s} \leq \sum_{\nu=N+1}^{\infty} v^{-\gamma / \tilde{\gamma}}
$$

and with $\gamma / \tilde{\gamma}=1+\delta / \tilde{\gamma}>1$, this tail is convergent and bounded above by $\varepsilon$ for sufficiently large $N$, independent of $n$ (compare also the proof on page 7 of [10]).

Next, we will show that unbounded subsequences do not contribute to the limit. For this, suppose that, for some fixed $\kappa,\left(\lambda_{n_{k}, \kappa}\right)_{k \in \mathbf{N}}$ is an unbounded subsequence, with $\lim _{k \rightarrow \infty} \lambda_{n_{k}, \kappa}=\infty$ and, by enlarging the subsequence if necessary, such that the sequence $\left(\lambda_{n, \kappa}\right)_{n \in\left(\mathbf{N}-\left\{n_{k}: k \in \mathbf{N}\right\}\right)}$ is either bounded or the empty set. Now, since $\lambda_{n_{k}, \kappa} \leq \lambda_{n_{k}, \mu}$ for $\mu \geq \kappa$, all sequences $\left(\lambda_{n_{k}, \mu}\right)_{k \in \mathbf{N}}$ for $\mu \geq \kappa$ tend to infinity as well. Observe now that the series

$$
D_{n_{k}}^{\geq \kappa}(s):=\sum_{\nu=\kappa}^{\infty} \frac{1}{\lambda_{n_{k}, v}^{s}}
$$

tends to the zero function as $n_{k}$ tends to infinity: $\lim _{n_{k} \rightarrow \infty} D_{n_{k}}^{\geq \kappa}(s)=\sum_{\nu=\kappa}^{\infty} \lim _{n_{k} \rightarrow \infty}$ $\frac{1}{\lambda_{n_{k}, v}^{s}}=0$, for $s$ real positive (hence for all $s$ by analytic continuation). In the above equation, we were allowed to interchange the order of the limits (in $n_{k}$ and the summation variable of $D_{n_{k}}$ ) since the series converge uniformly in $n_{k}$. Since we assume that $D_{n}$ is a convergent sequence of functions, it has the same limit as its the subsequence $D_{n_{k}}$.

Since we have now proven that unbounded subsequences do not contribute to the limit, we can assume that $\lambda_{n, v}$ is bounded in $n$, for all $v$, i.e., $\forall n \in \mathbf{N} \lambda_{n, v} \leq c_{\nu}$. Then, we can select a subsequence so that for all $v$, the $\operatorname{limit}_{k \rightarrow \infty} \lambda_{n_{k}, v}=\ell_{v}$ exists. Not to overload notation, we will momentarily relabel the convergent subsequence $\lambda_{n_{k}, v}$ as $\lambda_{n, v}$. In particular, $\lambda_{n, 1}$ converges to $\ell_{1}$. We will prove that $\lambda_{n, 1}$ converges to $\lambda_{1}$. Let us rewrite 


$$
D_{n}(s)=\frac{1}{\lambda_{n, 1}^{s}}\left(\sum_{\nu=1}^{\infty}\left(\frac{\lambda_{n, 1}}{\lambda_{n, v}}\right)^{s}\right)
$$

and

$$
D(s)=\frac{1}{\lambda_{1}^{s}}\left(\sum_{\nu=1}^{\infty}\left(\frac{\lambda_{1}}{\lambda_{\nu}}\right)^{s}\right) .
$$

We now assume that $s$ is an integer $s>\tilde{\gamma}$. Since $D_{n}(s) \rightarrow D(s)$, we have that

$$
\lim _{n \rightarrow \infty}\left(\frac{\lambda_{1}}{\lambda_{n, 1}}\right)^{s}=\frac{\sum_{\nu=1}^{\infty}\left(\frac{\lambda_{1}}{\lambda_{\nu}}\right)^{s}}{\lim _{n \rightarrow \infty} \sum_{\nu=1}^{\infty}\left(\frac{\lambda_{n, 1}}{\lambda_{n, v}}\right)^{s}} \leq \sum_{\nu=1}^{\infty}\left(\frac{\lambda_{1}}{\lambda_{\nu}}\right)^{s}
$$

For the last inequality, we have used the fact that $\lambda_{n, v}>0$ and that the denominator is $\geq 1$.

Set $\ell:=\lim _{n \rightarrow \infty} \lambda_{1} / \lambda_{n, 1}$. We now consider the limit as $s \rightarrow \infty$ (along the integers) in Eq. (3), to find $\lim _{s \rightarrow \infty} \ell^{s} \leq \#\left\{\lambda_{i}=\lambda_{1}\right\}$. With

$$
\lim _{s \rightarrow \infty} \ell^{s}= \begin{cases}1 & \text { if } \ell=1 \\ 0 & \text { if } \ell<1 \\ \infty & \text { if } \ell>1\end{cases}
$$

we find $\ell \leq 1$.

We also have the inequality

$$
\ell^{s}=\lim _{n \rightarrow \infty}\left(\frac{\lambda_{1}}{\lambda_{n, 1}}\right)^{s}=\frac{\sum_{\nu=1}^{\infty}\left(\frac{\lambda_{1}}{\lambda_{\nu}}\right)^{s}}{\lim _{n \rightarrow \infty} \sum_{\nu=1}^{\infty}\left(\frac{\lambda_{n, 1}}{\lambda_{n, v}}\right)^{s}} \geq \frac{1}{\sum_{\nu=1}^{\infty}\left(\frac{\ell_{1}}{\ell_{\nu}}\right)^{s}} .
$$

In the inequality, we have used that we can interchange limit and summation in the denominator, by uniform convergence. By taking the limit $s \rightarrow \infty$ (along the integers), we arrive at $\lim _{s \rightarrow \infty} \ell^{s} \geq \frac{1}{\#\left\{\ell_{n}=\ell_{1}\right\}}>0$. We conclude from all the above that $\ell=1$, and hence that

$$
1=\frac{\#\left\{\lambda_{i}=\lambda_{1}\right\}}{\#\left\{\ell_{n}=\ell_{1}\right\}} .
$$

Now recall that we have relabelled before, so that we have actually shown that every convergent subsequence $\left(\lambda_{n_{k}, 1}\right)_{k \in \mathbf{N}}$ of $\left(\lambda_{n, 1}\right)_{n \in \mathbf{N}}$ tends to some limit, and since $\ell=1$, all these subsequences converge to the same limit $\lambda_{1}$. Therefore, $\left(\lambda_{n, 1}\right)_{n \in \mathbf{N}}$ itself is convergent to $\lambda_{1}$. We conclude that in general (viz., before erasing all unbounded subsequences), every bounded subsequence of $\left(\lambda_{n}\right)_{n \in \mathbf{N}}$ converges to $\lambda_{1}$. 
We now use an inductive argument to treat the general term. Namely, consider the Dirichlet series $D_{n}^{\geq 2}(s):=D_{n}(s)-\lambda_{n, 1}^{-s}$ which (by what we have proven) converges to $D^{\geq 2}(s):=D(s)-\lambda_{1}^{-s}$. These are still sequences of Dirichlet series of the same form, but with first eigenvalues $\lambda_{n, 2}$ and $\lambda_{2}$. We can repeat the argument with this series, to conclude $\lambda_{n, 2} \rightarrow \lambda_{2}$, etc. This finishes the proof that (i) implies (ii).

Since we assume that $\Re(s)>\tilde{\gamma}$ is a common half-plane of convergence of all series $D_{n}(n=\emptyset, 1,2, \ldots)$, for any $\gamma>\tilde{\gamma}$, the sums $\sum_{\nu=1}^{\infty} \lambda_{n, \nu}^{-\gamma}$ converge, and hence, the sequences $\Lambda_{n}^{-\gamma}(n=\emptyset, 1,2, \ldots)$ belong to the Banach space $\ell^{1}$. We will now prove that $\Lambda_{n}^{-\gamma} \rightarrow \Lambda^{-\gamma}$ as elements of $\ell^{1}$.

To do so, we have to prove that for every $\epsilon>0$, there is an $n_{0} \in \mathbf{N}$ such that $n>n_{0}$ implies

$$
\sum_{\nu=1}^{\infty}\left|\frac{1}{\lambda_{n, \nu}^{\gamma}}-\frac{1}{\lambda_{\nu}^{\gamma}}\right| \leq \epsilon
$$

It is known that if $\left(a_{v}\right)$ is a sequence of positive real numbers so that $\sum_{v=1}^{\infty} a_{v}$ converges, then all of its "tails" tend to zero: $\lim _{N \rightarrow \infty} \sum_{\nu=N}^{\infty} a_{v}=0$. So, given an $\epsilon>0$, there is an $n_{0}$, which does not depend on $n$ (using the same $\gamma$ for all $n$ ), such that for $N \geq n_{0}$

$$
\sum_{\nu=N}^{\infty}\left|\frac{1}{\lambda_{n, \nu}^{\gamma}}\right|+\sum_{\nu=N}^{\infty}\left|\frac{1}{\lambda_{\nu}^{\gamma}}\right|<\epsilon / 2
$$

Therefore,

$$
\begin{aligned}
\sum_{\nu=1}^{\infty}\left|\frac{1}{\lambda_{n, \nu}^{\gamma}}-\frac{1}{\lambda_{\nu}^{\gamma}}\right| & \leq \sum_{\nu=1}^{N}\left|\frac{1}{\lambda_{n, \nu}^{\gamma}}-\frac{1}{\lambda_{\nu}^{\gamma}}\right|+\sum_{\nu=N}^{\infty}\left|\frac{1}{\lambda_{n, v}^{\gamma}}\right|+\sum_{\nu=N}^{\infty}\left|\frac{1}{\lambda_{\nu}^{\gamma}}\right| \\
& \leq \sum_{\nu=1}^{N} \frac{\left|\lambda_{\nu}^{\gamma}-\lambda_{n, v}^{\gamma}\right|}{\lambda_{n, \nu}^{\gamma} \lambda_{\nu}^{\gamma}}+\frac{\epsilon}{2} \\
& \leq \sum_{\nu=1}^{N} \frac{\left|\lambda_{\nu}^{\gamma}-\lambda_{n, v}^{\gamma}\right|}{C}+\frac{\epsilon}{2}
\end{aligned}
$$

where $0 \neq C=\inf _{1 \leq v \leq N}\left(\lambda_{1, v}^{\gamma} \lambda_{v}^{\gamma}\right) \leq\left(\lambda_{n, \nu}^{\gamma} \lambda_{v}^{\gamma}\right)$. Now, the finite number $(v=$ $1, \ldots, N)$ of sequences $\left(\lambda_{n, v}^{\gamma}\right)_{n \in \mathbf{N}}$ can be made to uniformly converge to $\lambda_{\nu}^{\gamma}$, that is, for every $\epsilon>0$, there is an $n_{1}$ such that $n>n_{1}$ implies $\left|\lambda_{v}^{\gamma}-\lambda_{n, v}^{\gamma}\right| \leq \frac{\epsilon C}{2 N}$ and inequality (5) gives us the desired result for all $n \geq \max \left\{n_{0}, n_{1}\right\}$.

This proves that (ii) implies (iii). Finally, if $\Lambda_{n}^{-\gamma}$ converges to $\Lambda^{-\gamma}$ for some $\gamma \in \mathbf{R}$, we have for every $s \in \mathbf{C}$ with $\Re(s)>\gamma$ that $\Lambda_{n}^{-s}$ converges to $\Lambda^{-s}$, and it follows easily that $D_{n}(s)$ converges to $D(s)$, pointwise in $s$. This proves that (iii) implies (i) and finishes the proof of the theorem.

For an application to the meaning of convergence in the cosmological distance from the previous section, suppose that $X=\left(X, g_{X}\right)$ and $Y=\left(Y, g_{Y}\right)$ are two 
isospectral, connected, smooth, closed Riemannian manifolds, i.e., suppose their Laplace-Beltrami operators $\Delta_{X}$ and $\Delta_{Y}$ have the same spectrum with multiplicities: $\Lambda_{X}=\Lambda_{Y}$ [16]. The spectrum $\Lambda_{X}$ is considered as a sequence $\left(\lambda_{\nu}\right)_{\nu=1}^{\infty}$ with $0 \leq \lambda_{1} \leq \lambda_{2} \leq \ldots$, with finite repetitions.

The identity theorem for Dirichlet series [10] shows that such isospectrality can also be described as the manifolds having the same zeta function: $\zeta_{X}=\zeta_{Y}$, where $\zeta_{X}(s):=$ $\operatorname{tr}\left(\Delta_{X}^{-s}\right)=\sum_{0 \neq \lambda \in \Lambda_{X}} \lambda^{-s}$, since connectedness implies that the zero eigenvalue has multiplicity one. The function $\zeta_{X}$ converges absolutely for $\Re(s)>d / 2$, where $d$ is the dimension of $X$ [16]. In this context, Theorem 3.2 says the following:

Proposition 3.5 Suppose $\left\{X_{n}\right\}_{n=1}^{\infty}$ is a sequence of connected, closed, smooth, Riemannian manifolds such that $d:=\sup \operatorname{dim} X_{n}$ is finite, and suppose that $X$ is another closed, smooth, Riemannian manifold. Then, the following statements are equivalent:

(i) For $\Re(s)>d / 2$, the functions $\zeta_{X_{n}}(s)$ converge pointwise to $\zeta_{X}(s)$;

(ii) For some $\gamma \in \mathbf{C}$ with $\operatorname{Re}(\gamma)>d / 2$, the sequence of eigenvalues $\Lambda_{X_{n}}^{-\gamma}$ converges to $\Lambda_{X}^{-\gamma}$ in $\ell^{1}$.

Remark 3.6 If the manifolds are closed and smooth and of odd dimension, but possibly disconnected, the equality $\zeta_{X}=\zeta_{Y}$ implies that also the multiplicity of the zero eigenvalue is equal for $X$ and $Y$, namely, it is minus the value at 0 of the analytic continuation of $\zeta_{X}([16], 5.2)$.

The circle of radius $r$ has $\Delta=-r^{2} \partial_{\theta}^{2}$ (with $\theta \in[0,2 \pi)$ the angle coordinate), spectrum $\lambda_{r, v}=r^{-2}\lceil\nu / 2\rceil^{2}$ and zeta function $\zeta_{r}(s)=r^{2 s} \zeta(2 s)$, where $\zeta$ is the Riemann zeta function. For varying $r \rightarrow r_{0}$, the convergence in the theorem happens for $\gamma>1 / 2$.

Already in the case of families of Riemannian manifolds, it can happen that $\left(\lambda_{n, \kappa}\right)$ has unbounded subsequences for some fixed $\kappa$; for example, a family of circles whose radius tends to zero. However, for fixed $\kappa$, we have bounds on the eigenvalues of the form ([3])

$$
C_{1} \sqrt[d]{\kappa^{2}} \leq \lambda_{n, \kappa} \leq \frac{C_{2}}{\operatorname{vol}\left(X_{n}\right)} \sqrt[d]{\kappa^{2}},
$$

where the constants $C_{i}$ depend on the dimension $d$, the diameter $D$, and a lower bound $R$ on the Ricci curvature of the manifolds under consideration. This implies that (at least if we fix the data $d, D$ and $R$, so we are in the Gromov precompact moduli space [9]) in unbounded subsequences, the volume should shrink to zero.

\section{Series with general coefficients}

In this section, we study what happens if we have pointwise convergence of general Dirichlet series in the sense of Eq. (1).

The previous case occurs when $\left\{\mu_{*}\right\}=\left\{\log \lambda_{*}\right\}$ and $a_{*}$ counts the multiplicities in $\left(\lambda_{*}\right)$. We start by discussing two special cases. 
4.1 (Taylor series) The case appears when $\mu_{n, v}=v$ for all $n=\emptyset, 1,2, \ldots$ In this case, we set $z=e^{-s}$ and we get a (pointwise) convergence of Taylor series $D_{n}(z)=\sum_{\nu \geq 0} a_{n, \nu} z^{\nu} \rightarrow D(z)=\sum_{\nu \geq 0} a_{\nu} z^{\nu}$. In this case, the individual series $D_{*}$ converge in $\Omega:=\left\{z>e^{-\gamma}\right\}$ to a holomorphic function (by assumption). Evaluation at zero gives $\lim _{n \rightarrow+\infty} a_{n, 0}=a_{0}$, and we can proceed by induction to conclude that $\lim _{n \rightarrow+\infty} a_{n, v}=a_{v}$ for all $v$.

Alternatively, one can use the representation of the coefficients by a complex contour integral to deduce the result "in a more complicated way". Namely, fix $\epsilon>0$, and let $n_{0}$ satisfy that $\left|D_{n}(z)-D(z)\right|<\epsilon$ for $n>n_{0}$, uniformly in $z \in K \subset \Omega$, where $K$ is a compact set. For a contour $C \subset K$ around $z=0$ (independent of $n$ ), we have

$$
\left|a_{n, v}-a_{v}\right| \leq \frac{1}{2 \pi} \int_{C}\left|D_{n}(z)-D(z)\right||z|^{-n-1} d z \leq \varepsilon
$$

4.2 (Constant exponents) The reason for providing this second proof is that it leads us to the next special case, in which we use the analogue of the integral representation for the coefficients for general Dirichlet series, also called Perron's formula. This formula gives a representation of the terms of a general Dirichlet series by integration over a vertical line in the complex plane, and since this integration domain, unlike the contour in the Taylor series proof, is not compact, we will need to work more to establish the result (or assume uniform convergence on an entire half-line, which seems too strong an assumption). This second special case occurs if $\mu_{n, v}$ is constant in $n$. Then we have the following result:

Theorem 4.3 Assume that $D_{n}(n=\emptyset, 1,2, \ldots)$ is a set of Dirichlet series that converge absolutely in a common half-plane $\Re(s)>\tilde{\gamma}$, and such that $D_{n}(s) \rightarrow D(s)$ converges pointwise there. Assume that $\mu_{n, v}=\mu_{v}$ is independent of $n$. Then, for every $n$, we have $\lim _{n \rightarrow+\infty} a_{n, v}=a_{v}$; actually, for $\sigma_{1}>\gamma$, we have a convergence of sequences $\left(a_{n, \nu} e^{-\sigma_{1} \mu_{\nu}}\right)_{\nu=1}^{\infty} \rightarrow\left(a_{\nu} e^{-\sigma_{1} \mu_{v}}\right)_{\nu=1}^{\infty}$ in $\ell^{\infty}$.

Proof Consider the difference $B_{n}(s):=D_{n}(s)-D(s)=\sum_{\nu \geq 1} b_{n, \nu} e^{-s \mu_{\nu}}$, where $b_{n, v}:=a_{n, v}-a_{v}$. According to Theorem I.3.1 in [13], we have the following integral representation for every $n$ and every fixed $v$ :

$$
\begin{aligned}
\left|b_{n, \nu} e^{-\sigma_{1} \mu_{v}}\right| & =\left|\lim _{T \rightarrow \infty} \frac{1}{T} \int_{0}^{T} B_{n}\left(\sigma_{1}+i t\right) e^{\mu_{v} i t} d t\right| \\
& \leq \lim _{T \rightarrow \infty} \frac{1}{T} \int_{0}^{T}\left|B_{n}\left(\sigma_{1}+i t\right)\right| d t
\end{aligned}
$$

We could finish the proof here by assuming that $D_{n}$ converges uniformly to $D$ on the entire line $\mathfrak{R}(s)=\sigma_{1}$. However, we can avoid this (strong) hypothesis by proving the following lemma:

Lemma 4.4 For every $\epsilon>0$, there is a $t_{0} \in \mathbf{R}$ such that

$$
\forall t \in \mathbf{R}, \forall n,\left|B_{n}\left(\sigma_{1}+i t\right)\right| \leq \epsilon+\left|B_{n}\left(\sigma_{1}+i t_{0}\right)\right|
$$


We are then finished with the proof of Theorem 4.3, since now, given any $\epsilon>0$, the pointwise convergence at $t_{0}$ implies that there exists $n_{0}$ such that for all $n>n_{0}$,

$$
\left|B_{n}\left(\sigma_{1}+i t\right)\right| \leq \epsilon+\left|B_{n}\left(t_{0}\right)\right| \leq 2 \epsilon
$$

and then, the above inequality becomes $\left|b_{n, v} e^{-\sigma_{1} \mu_{v}}\right| \leq 2 \epsilon$. Since $\sigma_{1}$ and $v$ are fixed, $e^{-\sigma_{1} \mu_{v}}$ is a non-zero constant, and this proves that $b_{n, v} \rightarrow 0$ as $n \rightarrow+\infty$. Since the $\epsilon$-bound holds uniformly in $v$, we do find the $\ell^{\infty}$ convergence as stated.

Proof of Lemma 4.4 Since the series $D_{n}$ are absolutely convergent on a common halfplane, their sequences of tails tend to zero uniformly in $n$, that is, for every $\epsilon>0$, there is an $N$, that is independent of $n$, such that

$$
\sum_{\nu=N+1}^{\infty}\left|a_{n, \nu} e^{-s \mu_{\nu}}\right|+\sum_{\nu=N+1}^{\infty}\left|a_{\nu} e^{-s \mu_{\nu}}\right|<\epsilon .
$$

Hence,

$$
\left|B_{n}(s)\right| \leq\left|B_{n}^{\leq N}(s)+D_{n}^{>N}(s)-D^{>N}(s)\right| \leq\left|B_{n}^{\leq N}(s)\right|+\epsilon
$$

We will now estimate the sum of the first $N$ terms on a vertical line $\Re(s)=\sigma_{1}$. Consider the function $f: \mathbf{R} \rightarrow\left(S^{1}\right)^{N}$ given by $t \mapsto\left(e^{i t \mu_{1}}, \ldots, e^{i t \mu_{N}}\right)$ and the function $F:\left(S^{1}\right)^{N} \rightarrow \mathbf{C}$ sending $\left(P_{1}, \ldots, P_{N}\right) \mapsto \sum_{v=1}^{N} e^{-\sigma_{1} \mu_{v}} P_{v}$. The function $F$ is continuous on a compact set; therefore, it attains a maximal value $M$ at a point $A^{0}:=\left(P_{1}^{0}, \ldots, P_{N}^{0}\right)$.

Lemma 4.5 There exists $t_{0} \in \mathbf{R}$ such that all the numbers $\left\{t_{0} \mu_{\nu}\right\}_{\nu=1}^{\infty}$ are irrational.

Proof The set of multiples $\{b \rho\}$ of a given real number $\rho \in \mathbf{R}$ such that $b \rho \in \mathbf{Q}$ is just $\frac{1}{\rho} \mathbf{Q}$ and this set is denumerable. A denumerable union of denumerable sets cannot exhaust the set of reals and the result follows.

This proves that the set $f(\mathbf{R})$ is dense in $\left(S^{1}\right)^{N}$. Therefore, for every $\delta>0$, there exists $t_{0} \in \mathbf{R}$ such that $\left|f\left(t_{0}\right)-A^{0}\right| \leq \delta$, and hence, since $F$ is continuous,

$$
\left|B_{n}^{\leq N}\left(t_{0}\right)-M\right|=\left|F\left(f\left(t_{0}\right)\right)-F\left(A^{0}\right)\right|<\epsilon .
$$

Since $M$ is the maximum, for all $t \in \mathbf{R}$, we have

$$
\left|B_{n}^{\leq N}\left(\sigma_{1}+i t\right)\right| \leq M \leq\left|B_{n}^{\leq N}\left(\sigma_{1}+i t_{0}\right)\right|+\left|B_{n}^{\leq N}\left(\sigma_{1}+i t_{0}\right)-M\right| .
$$

By Eqs. (6), (7) and (8) we now have

$$
\begin{aligned}
\left|B_{n}\left(\sigma_{1}+i t\right)\right| & \leq \epsilon+\left|B_{n}^{\leq N}\left(\sigma_{1}+i t\right)\right| \\
& \leq \epsilon+\left|B_{n}^{\leq N}\left(\sigma_{1}+i t_{0}\right)\right|+\left|B_{n}^{\leq N}\left(\sigma_{1}+i t_{0}\right)-M\right| \\
& \leq 2 \epsilon+\left|B_{n}^{\leq N}\left(\sigma_{1}+i t_{0}\right)\right|,
\end{aligned}
$$

and this finishes the proof of lemma and of the Theorem 4.4. 
4.6 We give an application on how to deduce convergence of eigenfunctions from a spectral convergence, following a method introduced in [7], 2.5-2.7. Let $X$ denote a closed, smooth, Riemannian manifold and let $a \in C^{\infty}(X)$ denote a smooth function. Define a generalized Dirichlet series by $\zeta_{X, a}:=\operatorname{tr}\left(a \Delta_{X}^{-s}\right)$, cf. [7]. Then,

$$
\zeta_{X, a}=\sum_{0 \neq \lambda \in \Lambda_{X}} \frac{1}{\lambda^{s}} \cdot \int_{X} a \sigma_{X, \lambda}
$$

where $\Lambda_{X}$ is the spectrum without multiplicities, and $\sigma_{X, \lambda}:=\sum_{\lambda \dashv \Psi}|\Psi|^{2}$ is the sum of the elements $\Psi$ of an orthonormal basis of eigenfunctions that belong to the eigenvalue $\lambda$.

Now assume that we have a compact manifold $X$ and a family $\left\{g_{r}\right\}(r \in \mathbf{R})$ of isospectral metrics with simple eigenvalues on $X$ (cf. Gordon and Wilson [8] for the existence of such families). Denote by $\Psi_{r, \lambda}$ the normalized real eigenfunction for the metric $g_{r}$ corresponding to the eigenvalue $\lambda$. If all zeta functions converge in the sense that

$$
\zeta_{X, g_{r}, a} \rightarrow \zeta_{X, g_{0}, a} \text { for all } a
$$

pointwise, for all $a$, then we find from the above result that $\int a \Psi_{r, \lambda}^{2} d \mu_{r} \rightarrow \int a \Psi_{0, \lambda}^{2} d \mu_{0}$ for all functions $a \in C^{\infty}(X)$, where $\mu_{r}$ is the measure belonging to the metric $g_{r}$, so a weak convergence of measures $\Psi_{r, \lambda}^{2} d \mu_{r} \rightarrow \Psi_{0, \lambda}^{2} d \mu_{0}$. Taking residues at $\operatorname{dim}(X) / 2$ in (9) for $a=1$, we find that the volume of $\left(X, g_{r}\right)$ is constant in $r$, and then taking residues for general $a$, we find that for all $a \in C^{\infty}(X), \int a d \mu_{r} \rightarrow \int a d \mu_{0}$ : there is a weak convergence of measures $\mu_{r} \rightarrow \mu_{0}$. Hence, we have a (pointwise) convergence of squared eigenfunctions: $\Psi_{r, \lambda}^{2} \rightarrow \Psi_{0, \lambda}^{2}$.

\section{General case}

Finally, in the most general case of varying coefficients and varying exponents, we prove a theorem about accumulation points. First, we do some preparation.

Definition 5.1 For a fixed strictly positive real function $g$, define for a real function $f$, the $g$-sup norm as

$$
\|f\|_{\infty, g}:=\sup _{x \in \mathbf{R}}\left|\frac{f(x)}{g(x)}\right|,
$$

when it is defined. We say that a sequence of functions $\left\{f_{n}\right\}$ converges multiplicatively to a real function $f$ if there exists a strictly positive real function $g$ that is integrable with respect to the multiplicative Haar measure on $\mathbf{R}^{*}$ (i.e., such that $\int_{\mathbf{R}} g(x) \frac{d x}{|x|}<+\infty$ ), such that $\left\|f_{n}(x)-f(x)\right\|_{\infty, g} \rightarrow 0$ for $n \rightarrow+\infty$. 
Definition 5.2 For $f$, a complex function defined for $\Re(s)=c$, and $x \in \mathbf{R}$, denote by

$$
I_{x}^{c}(f):=\int_{\mathfrak{R}(s)=c} f(s) e^{x s} \frac{d s}{s} .
$$

The relevance of this integral for the theory of Dirichlet series lies in the following formula of Perron [10, th. 13]: if $D(s)=\sum_{v \geq 1} a_{\nu} e^{-s \mu_{\nu}}$ is convergent for $s=\beta+i \gamma$ and $c>0, c>\beta, x \in \mathbf{R}, x \geq \beta, n \in \mathbf{N}$ such that $\mu_{n} \leq x<\mu_{n+1}$, then

$$
\sum_{\nu=1}^{n} a_{v}=\frac{1}{2 \pi i} I_{x}^{c}(D),
$$

with the convention that the last summand on the left-hand side is multiplied by $1 / 2$ if $x$ equals $\mu_{n}$.

Since $I_{x}^{c}(D)$ does not depend on $c$ once it satisfies the conditions for Perron's formula, we will now write $I_{x}(D)$ for $I_{x}^{c}(D)$ with any suitable $c$.

Lemma 5.3 If $\left\{f_{n}(c+i t)\right\}$ converges multiplicatively to $f(c+i t)$ in $t$, then for all $x \in \mathbf{R}$,

$$
\lim _{n} I_{x}\left(f_{n}\right)=I_{x}\left(\lim _{n} f_{n}\right)=I_{x}(f) .
$$

Proof We have

$$
\begin{aligned}
\left|I_{x}\left(f_{n}\right)-I_{x}(f)\right| & \leq \int_{\mathbf{R}}\left|\frac{f_{n}(c+i t)-f(c+i t)}{c+i t} e^{x(c+i t)}\right| d t \\
& \leq e^{c x}\left(\int_{\mathbf{R}} \frac{g(t)}{\sqrt{c^{2}+t^{2}}} d t\right) \cdot\left\|f_{n}(c+i t)-f(c+i t)\right\|_{\infty, g} \\
& \leq e^{c x}\left(\int_{\mathbf{R}} \frac{g(t)}{|t|} d t\right) \cdot\left\|f_{n}(c+i t)-f(c+i t)\right\|_{\infty, g} \\
& \leq C \varepsilon
\end{aligned}
$$

with $C=e^{c x}\left(\int_{\mathbf{R}} \frac{g(t)}{|t|} d t\right)$ finite constant, for $n$ sufficiently large. This proves the desired result.

Before stating the main result of this section, we need to introduce some notation:

Notation 5.4 Write $\mu_{n, j}=\log \lambda_{n, j}$, and assume that all sequences $\left(\lambda_{n, j}\right)_{n=1}^{\infty}$ are bounded. Let $\ell_{i}^{(j)}, i \in I_{j}$ be the accumulation points of sequence $\left(\lambda_{n, j}\right)_{n=1}^{\infty}$.

We consider a subsequence $n_{k}$ such that for all $j \lim _{n_{k} \rightarrow \infty} \lambda_{n_{k}, j}=\ell_{i_{j}}^{(j)}$ for a selection $i_{j} \in I_{j}$. Notice that the sequences $\left(\lambda_{n_{k}, j}\right)_{k=1}^{\infty}$ and $\left(\lambda_{n_{k}, j+1}\right)_{k=1}^{\infty}$ satisfy $\lambda_{n_{k}, j}<\lambda_{n_{k}, j+1}$, but they can tend to the same accumulation point. 
For the infinite vector of convergent sequences $\left(\left(\lambda_{n_{k}, j}\right)_{k=1}^{\infty}\right)_{j \geq 1}$ converging to the infinite vector $\left(\ell_{i_{j}}^{(j)}\right)_{j \geq 1}$, we consider the sequence $m_{1}, m_{2}, \ldots$, such that

$$
\ell_{i_{1}}^{(1)}=\ell_{i_{2}}^{(2)}=\cdots=\ell_{i_{m_{1}}}^{\left(m_{1}\right)}, \ell_{i_{m_{1}+1}}^{\left(m_{1}+1\right)}=\ell_{i_{m_{1}+2}}^{\left(m_{1}+2\right)}=\cdots=\ell_{i_{m_{2}}}^{\left(m_{2}\right)} \text {, etc. }
$$
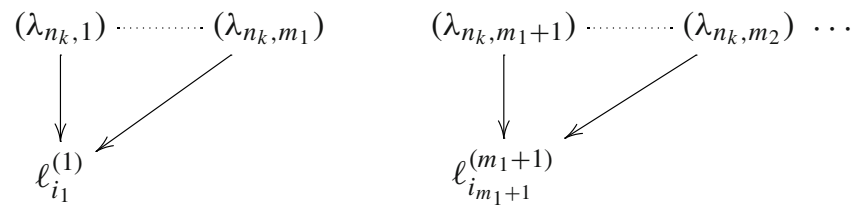

Theorem 5.5 We use the notation of (5.4). Assume that $D_{n}$ converges multiplicatively to $D(s)=\sum_{j \geq 1} a_{j} e^{-s \log \lambda_{j}}$. Then, $\lambda_{j}$ are accumulation points for some sequence $\left(\lambda_{n, j^{\prime}}\right)_{n=1}^{\infty}$.

Consider the set of subsequences $\left(\left(\lambda_{n, j}\right)_{n=1}^{\infty}\right)_{j \geq 1}$ converging to the infinite vector $\left(\ell_{i_{j}}^{(j)}\right)_{j \geq 1}$. Suppose that the sequences $\left(\lambda_{n_{k}, j}\right)_{n=1}^{\infty}$ for $j=m_{\mu}+1, \ldots, m_{\mu+1}$ converge to $\ell$. Set

$$
A_{n_{k}}^{(\mu)}:=\sum_{j=m_{\mu}+1}^{m_{\mu+1}} a_{n_{k}, j}, \text { for } \mu \geq 0
$$

Then,

$$
\lim A_{n_{k}}^{(\mu)}= \begin{cases}a_{i} & \text { if } \ell=\lambda_{i} \\ 0 & \text { otherwise }\end{cases}
$$

Proof Assume that the set of subsequences $\left(\left(\lambda_{n_{k}, j}\right)_{k=1}^{\infty}\right)_{j \geq 1}$ converges to the set of accumulation points $\left(\ell_{i}^{(j)}\right)$.

Consider the first eigenvalue $\lambda_{1}$ of $D$. If $\ell$ is the first element in the set $\ell_{i}^{(j)}$ that is smaller than $\lambda_{1}$, then by choosing $x$ such that $\ell<x<\lambda_{1}$, by Perron's formula, we have that $I_{x}\left(D_{n_{k}}\right)=\sum_{j=1}^{m_{1}} a_{n_{k}, j}=A_{n_{k}}^{(0)}$ should tend to $I_{x}(D)=0$ since $x<$ $\lambda_{1}$. This proves that $A_{n_{k}}^{(0)}$ tends to zero as desired. We proceed, now, to the next accumulation point that is smaller than $\lambda_{1}$, and by the same argument, we prove that $\lim _{n_{k} \rightarrow \infty} \sum_{j=1}^{m_{2}} a_{n_{k}, j}=0$. Then, since the limit of the sum of the first $m_{1}$ terms tends to zero, we have that

$$
\lim _{n_{k} \rightarrow \infty} \sum_{j=m_{1}+1}^{m_{2}} a_{n_{k}, j}=0,
$$

and so, the desired result is proved for all $\ell<\lambda_{1}$. 
We will prove now that $\lambda_{1}$ is an accumulation point. Indeed, for sufficiently small $\epsilon>0$, the quantity $I_{\lambda_{1}-\epsilon}(D)-I_{\lambda_{1}+\epsilon}(D)=a_{1} \neq 0$. Using the above equation and lemma 5.3, we obtain

$$
\lim _{n_{k} \rightarrow \infty}\left(I_{\lambda_{1}-\epsilon}\left(D_{n_{k}}\right)-I_{\lambda_{1}+\epsilon}\left(D_{n_{k}}\right)\right)=a_{1}=\lim \sum_{\lambda_{1}-\epsilon<\lambda_{n_{k}, j}<\lambda_{1}+\epsilon} a_{n_{k}, j} .
$$

So, by taking small $\epsilon$, we can find a subsequence tending to $\lambda_{1}$, so $\lambda_{1}$ is one of the accumulation points of the sequence $\left(\left(\lambda_{n_{k}, j}\right)_{k=1}^{\infty}\right)_{j \geq 1}$. Notice that, as a result, Eq. (10) is also proved.

We continue the proof by induction by taking $\ell$ to be between $\lambda_{1}$ and $\lambda_{2}$, so that the corresponding sum tends to zero; then, we take $\ell$ to be $\lambda_{2}$, then between $\lambda_{2}$ and $\lambda_{3}$ etc.

\section{Relation with Laplace-Stieltjes transform}

The notion of Dirichlet series and Laplace transforms can be unified in terms of the Riemann-Stieltjes integrals (Widder [20], compare [2]).

Definition 6.1 Suppose $\omega \geq 0$ is a real number.

1. The space $\operatorname{Lip}_{\omega}$ is defined as the set of functions $F: \mathbf{R}_{\geq 0} \rightarrow \mathbf{R}$ with bounded norm

$$
\|F\|_{\text {Lip }, \omega}:=\sup _{0 \leq s<t} \frac{|F(t)-F(s)|}{(t-s) e^{\omega t}}<\infty .
$$

2. The space $\operatorname{Wid}_{\omega}$ is defined as the space of smooth function $(\omega, \infty) \rightarrow \mathbf{R}$ with bounded norm

$$
\|D\|_{\text {Wid, } \omega}:=\sup _{\substack{s>\omega \\ k \in \mathbf{N}}} \frac{(s-\omega)^{k+1}}{k !}\left|\frac{d^{k} D}{d s^{k}}(s)\right|<\infty .
$$

The main result is now that the so-called Laplace-Stieltjes transform $F \mapsto$ $\int_{0}^{\infty} e^{-s t} d F(t)$ induces an isometric isomorphism $\operatorname{Lip}_{\omega} \rightarrow \operatorname{Wid}_{\omega}$ ([2], Thm. 2.4.1).

Widder ([20], Theorems 11.212.4) proved that a Dirichlet series of the form $D(s)=$ $\sum_{v} a_{\nu} e^{-s \mu_{v}}$ convergent for $\Re(s)>\omega$ is in the space $\operatorname{Wid}_{\omega}$. Also, such $D$ is the Laplace-Stieltjes transform of $F(t)=\sum_{\nu=0}^{\infty} a_{\nu} H\left(t-\mu_{\nu}\right)$, where $H$ is the Heaviside step function. Thus, we immediately conclude the following:

Theorem 6.2 Suppose $D_{n}(s)=\sum_{v \geq 1} a_{n, v} e^{-s \mu_{n, v}}$ is a sequence of Dirichlet series each converging absolutely in a common half-plane $\Re(s)>\gamma$; then for any $\omega>\gamma$, $D_{n}$ converges to a Dirichlet series $D(s)=\sum_{\nu \geq 1} a_{\nu} e^{-s \mu_{\nu}}$ in $\operatorname{Wid}_{\omega}$-norm if and only if $\sum_{\nu=0}^{\infty}\left(a_{n, \nu} H\left(t-\mu_{n, \nu}\right)-a_{\nu} H\left(t-\mu_{\nu}\right)\right) \rightarrow 0$ in $\operatorname{Lip}_{\omega^{-n o r m}}$.

It would be interesting to deduce Theorems 4.3 and 5.5 from Theorem 6.2. 
Open Access This article is distributed under the terms of the Creative Commons Attribution 4.0 International License (http://creativecommons.org/licenses/by/4.0/), which permits unrestricted use, distribution, and reproduction in any medium, provided you give appropriate credit to the original author(s) and the source, provide a link to the Creative Commons license, and indicate if changes were made.

\section{References}

1. Aasen, D., Bhamre, T., Kempf, A.: Shape from sound: toward new tools for quantum gravity. Phys. Rev. Lett. 110(12), 121301 (2013)

2. Arendt, W., Batty, C.J.K., Hieber, M., Neubrander, F.: Vector-valued Laplace transforms and Cauchy problems. In: Monographs in mathematics, vol. 96. Birkhäuser, Basel (2001)

3. Bérard, P., Besson, G., Gallot, S.: Embedding Riemannian manifolds by their heat kernel. Geom. Funct. Anal. 4(4), 373-398 (1994)

4. Buchert, T., Ehlers, J.: Averaging inhomogeneous Newtonian cosmologies. Astron. Astrophys. 320, 1-7 (1997)

5. Connes, A.: Gravity coupled with matter and the foundation of non-commutative geometry. Commun. Math. Phys. 182(1), 155-176 (1996)

6. Conway, J.B.: Functions of one complex variable. In: Graduate Texts in Mathematics, 2nd ed., vol. 11, Springer, New York (1978)

7. Cornelissen, G., de Jong, J.W.: The spectral length of a map between Riemannian manifolds. J. Noncommut. Geom. 6(4), 721-748 (2012)

8. Gordon, C.S., Wilson, E.N.: Continuous families of isospectral Riemannian metrics which are not locally isometric. J. Differ. Geom. 47(3), 504-529 (1997)

9. Gromov, M.: Metric structures for Riemannian and non-Riemannian spaces. In: Modern Birkhäuser Classics. Birkhäuser Boston Inc., Boston (2007)

10. Hardy, G.H., Riesz, M.: The general theory of Dirichlet's series. In: Cambridge Tracts in Mathematics and Mathematical Physics, No. 18, Stechert-Hafner Inc, New York (1964)

11. Hobson, M.P., Jaffe, A.H., Liddle, A.R., Mukherjee, P., Parkinson, D. (eds.): Bayesian Methods in Cosmology. Cambridge University Press, Cambridge (2010)

12. Landi, G., Rovelli, C.: General relativity in terms of Dirac eigenvalues. Phys. Rev. Lett. 78(16), 30513054 (1997)

13. Mandelbrojt, S.: Séries de Dirichlet. Principes et méthodes, Monographies Internationales de Mathématiques Modernes, vol. 11. Gauthier-Villars, Paris (1969)

14. Marcolli, M., Pierpaoli, E., Teh, K.: The spectral action and cosmic topology. Commun. Math. Phys. 304, 125-174 (2011)

15. Niarchou, A., Jaffe, A.: Imprints of spherical non-trivial topologies on the CMB. Phys. Rev. Lett. 99, 081302 (2007)

16. Rosenberg, S.: The Laplacian on a Riemannian manifold, London Mathematical Society Student Texts, vol. 31. Cambridge University Press, Cambridge (1997)

17. Seriu, M.: Spectral representation of the spacetime structure: the "distance" between universes with different topologies. Phys. Rev. D (3) 53(12), 6902-6920 (1996)

18. Seriu, M.: Spectral representation and the averaging problem in cosmology. General. Relariv. Gravit 32, 1473-1485 (2000)

19. 't Hooft, G.: The fundamental nature of space and time. In: Oriti, D. (ed.) Approaches to Quantum Gravity, pp. 13-25. Cambridge University Press, Cambridge (2009)

20. Widder, D.V.: A generalization of Dirichlet's series and of Laplace's integrals by means of a Stieltjes integral. Trans. Am. Math. Soc. 31(4), 694-743 (1929) 